\title{
Feel between the Lines: Implied Emotion in Sentence Comprehension
}

\author{
Vicky Tzuyin Lai ${ }^{1,2}$, Roel M. Willems ${ }^{1,3}$, and Peter Hagoort ${ }^{1,3}$
}

\begin{abstract}
This study investigated the brain regions for the comprehension of implied emotion in sentences. Participants read negative sentences without negative words, for example, "The boy fell asleep and never woke up again," and their neutral counterparts "The boy stood up and grabbed his bag." This kind of negative sentence allows us to examine implied emotion derived at the sentence level, without associative emotion coming from word retrieval. We found that implied emotion in sentences, relative to neutral sentences, led to activation in
\end{abstract}

\section{INTRODUCTION}

We read, and oftentimes we feel emotional when we read. How does the brain gather emotion from language during reading? There are several ways that emotion can be induced by language. One straightforward way is through word associations, including words associated with emotions (e.g., sad, happy) and words associated with emotionally significant events (e.g., assassinate). Past research has shown that these words lead to activations in a range of brain regions, including amygdala, parahippocampal and lingual gyrus, cingulate cortex, and visual areas (Moseley, Carota, Hauk, Mohr, \& Pulvermuller, 2012; Herbert et al., 2009; Isenberg et al., 1999). Another way language induces emotion is less straightforward. In our daily life, we understand not only emotion explicitly stated in words but also emotion implied by a series of neutral words. For example, in the sentence "The boy fell asleep and never woke up again," the unpleasant emotion is not explicitly stated, but the sentence triggers negative feelings-that is, the emotion is implied. This study is interested in how the brain supports the computation of implied emotion in language. Specifically, we ask two questions: First, would implied emotion engage brain regions involved in explicit, associative emotion? Second, would implied emotion influence areas supportive of language comprehension?

In what follows, we first review the regions commonly reported for emotion and then for language. Most emotion

\footnotetext{
${ }^{1}$ Max Planck Institute for Psycholinguistics, Nijmegen, the Netherlands, ${ }^{2}$ University of South Carolina, ${ }^{3}$ Donders Institute for Brain, Cognition and Behaviour, Nijmegen, the Netherlands

some emotion-related areas, including the medial prefrontal cortex, the amygdala, and the insula, as well as certain languagerelated areas, including the inferior frontal gyrus, which has been implicated in combinatorial processing. These results suggest that the emotional network involved in implied emotion is intricately related to the network for combinatorial processing in language, supporting the view that sentence meaning is more than simply concatenating the meanings of its lexical building blocks. studies used pictures, and based on the pictorial modality, the regions commonly reported for emotion include the amygdala, the medial prefrontal cortex (mPFC), the anterior cingulate gyrus, and the insula (Luan Phan, Wager, Taylor, \& Liberzon, 2004). Different functional roles have been proposed for these regions. Amygdala responds to emotionally arousing information, including both negative emotions such as fear and threat (Davis \& Whalen, 2001) and positive emotions such as happiness (Hamann \& Mao, 2002). The $\mathrm{mPFC}$ has been argued to play a general role for many kinds of emotions across various stimulus formats (Lane, Reiman, Ahern, Schwartz, \& Davidson, 1997) and is related to the cognitive generation and regulation of affect (Teasdale et al., 1999), as well as introspective mental activities (Gusnard, Akbudak, Shulman, \& Raichle, 2001). Moreover, this region and its subregions have been discussed in the research contexts of social cognition and theory of mind. In particular, the anterior region of the rostral medial frontal cortex has been implicated in the evaluation of one's own emotion, the perception and judgment about other people, and the ability to represent another person's psychological perspective (Amodio \& Frith, 2006). The last function is also known as "mentalizing"- that is, the ability to predict someone else's mental state based on their own belief and the emotional impact of the belief (Frith \& Frith, 2006). Closely related to the $\mathrm{mPFC} /$ medial frontal cortex is the ACC. It has been proposed that the dorsal ACC and $\mathrm{mPFC}$ are involved in cognitively generated emotional responses, whereas the ventral portions of ACC and mPFC play more of a regulatory role for emotional responses (Etkin, Egner, \& Kalisch, 2011). The insula is another region that is commonly observed in emotion studies. 
The insula has been implicated for internally generated emotion, but not so much for externally induced emotion via pictures (Reiman et al., 1997). One proposal suggested that the insula is a somatic marker that maps our bodily states to the associated emotional experiences (Damasio, Everitt, \& Bishop, 1996; but see Dunn, Dalgleish, \& Lawrence, 2006). Another proposal suggested that the insula plays an important role in social emotion, such as empathy and compassion (Lamm \& Singer, 2010), and serves as an interface that integrates several functional systems involved in affect, sensory-motor processing, and cognition (Chang, Yarkoni, Khaw, \& Sanfey, 2013).

Words differ from pictures in that words represent a type of emotional event indirectly, whereas pictures depict the specifics, representing a token of the emotional event of interest directly. Several studies have examined emotional words, but to date there is no clear consensus as to which regions are definitely involved. Amygdala involvement has been reported in some (Strange, Henson, Friston, \& Dolan, 2000; Isenberg et al., 1999), but not others (Kuchinke et al., 2005; Cato et al., 2004). Within those that found amygdala involvement, they also found that amygdala modulated the visual processing of emotionally valenced words in the occipital cortex (e.g., Tabert et al., 2001). In those that did not show amygdalar responses, some observed activation in the posterior cingulate cortex instead (Kuchinke et al., 2005; Maddock, Garrett, \& Buonocore, 2003), whereas others observed activation in the left inferior frontal cortex instead (Nakic, Smith, Busis, Vythilingam, \& Blair, 2006). These mixed findings may be driven by task: The more evaluative the task is, the more likely it is to find amygdala activation.

At the sentence level, only a couple of studies have examined emotion and explicit emotion. In a lesion study, Adolphs, Russell, and Tranel (1999) examined the functional role of the amygdala in a rare patient with bilateral amygdala damage. The patient was presented with sentences such as "Sally waved her hands in the air and yelled for belp, as the boat was sinking." The patient was impaired in judging how emotionally arousing these sentences were but was intact in judging whether the sentences were pleasant or unpleasant. Moll, de OliveiraSouza, Bramati, and Grafman (2002) examined moral statements. Relevant to this study, within their nonmoral sentences, enhanced activities were observed for the unpleasant relative to the neutral statements in the left amygdala, orbital frontal cortex, lingual gyri, and fusiform areas. Holt et al. (2011) examined the comprehension of emotional descriptions in schizophrenia and healthy controls. Negative relative to neutral sentences in healthy participants showed activations in the right posterior cingulate gyrus, right precuneus, and left posterior cingulate gyrus. Finally, Willems, Clevis, and Hagoort (2011) found that emotional sentences compared to nonemotional sentences showed more activation in the left insula, the left temporal pole, and the anterior part of the left inferior frontal region.
Holt et al. (2011), Moll et al. (2002), and Adolphs et al. (1999) used emotional sentences that contain emotional words (e.g., yelled, sinking), providing word associations with explicit emotion. In strong contrast and crucially, this study used emotional sentences without emotionally valenced words, aiming at investigating the neurobiological underpinnings of the computation of implied emotion. We suggest that implied emotion in sentences require "combinatorial processing." Combinatorial processing in language refers to the process in which words are combined into multiword representations to give rise to sentential meaning beyond the meaning of single words (Hagoort, 2013; Humphries, Binder, Medler, \& Liebenthal, 2007; Hagoort, 2005). Combinatorial processing is assumed to happen at multiple levels, such as sound, syntax, and meaning (Jackendoff, 2002). To test emotion generated by combinatorial processing and combinatorial processing alone, we designed negative sentences that contain no negative words (e.g., "The boy fell asleep and never woke up again") and their neutral counterparts ("The boy stood up and grabbed his bag"). In both the negative and neutral sentences, none of the individual lexical items are associated with negative feelings, but the negative sentence as a whole triggers negative feelings, whereas the neutral sentence does not.

Most language studies examining combinatorial processing of this kind reported enhanced activations in the regions of the left inferior frontal gyrus (LIFG), the anterior and posterior temporal regions, and the angular gyrus (Brennan \& Pylkkänen, 2012; Rodd, Johnsrude, \& Davis, 2012; Bemis \& Pylkkänen, 2011; Graves, Binder, Desai, Conant, \& Seidenberg, 2010; Snijders et al., 2009; Humphries et al., 2007; Rodd, Davis, \& Johnsrude, 2005). Activations in the LIFG have been reliably found for various types of stimuli, ranging from simple combinations such as two-word compounds (e.g., meaningful "flower girl" vs. reversed "girl flower"; Graves et al., 2010) to complex combinations in multiword utterances (e.g., sentences with ambiguous words "The shell was fired towards the tank" vs. sentences with unambiguous words "Her secrets were written in her diary"; Rodd et al., 2005). Several hypotheses have been put forth for the functional role of LIFG during language processing: The LIFG may contribute to the parsing and reparsing of syntactic information (Novick, Trueswell, \& Thompson-Schill, 2005), the reinterpretation of semantic information (Rodd et al., 2012), and the integration of information that is not stored in longterm memory (Hagoort, 2005, 2013; Willems, Ozyurek, \& Hagoort, 2007). The LIFG can be anatomically subdivided into pars orbitalis, pars opercularis, and pars triangularis, each of which has been implicated in the subprocesses in language processing (Hagoort, 2005). Specifically, the dorsal parts of the LIFG (pars triangularis and pars opercularis) are implicated in syntactic processing, and the ventral parts of the LIFG (pars triangularis and pars orbitalis) are implicated in semantic processing (Hagoort \& Indefrey, 
2014). In addition, pars opercularis has been shown to be involved in phonological processing (Hagoort, 2013).

Not all language theories would support the claim that emotion can be derived from combinatorial language processing. According to a strong version of the embodied theories, emotional language is grounded in the emotional states of the body, and simulating those is a prerequisite for language comprehension (Glenberg, Havas, Becker, \& Rinck, 2005). In support of this view, it has been shown that emotional words such as weep activate motor areas, suggesting that emotional word meaning is understood through simulating emotion-expressing actions previously experienced (Moseley et al., 2012). However, most of these studies examined emotion associated with words, not emotion derived from word combinations in sentences. It is not clear if the emotionby-simulation theories can account for implied emotion at the sentence level, which cannot come from word retrieval, but must stem from combinatorial processing in sentences. In addition, some studies (e.g., Moseley et al., 2012; Kuchinke et al., 2005) also found activations for emotional words in classic language regions such as inferior frontal gyrus (IFG), typically implicated for combinatorial language processing.

This study examines the comprehension of implied emotion in sentences. To this end, we measured hemodynamic changes while participants read negative sentences without negative words (e.g., The boy fell asleep and never woke up again), negative words (e.g., coma), and negative pictures (e.g., a picture of a man being bullied), along with their neutral counterparts. We added the conditions of words and pictures because, although our primary interest is sentences, we are also interested in the commonalities and differences between words, pictures, and sentences. We predict that implied emotion, as revealed by subtracting the neutral sentences from the negative sentences, will likely lead to activation in the MPFC, the amygdala, and the insula, because implied emotion tested here can be viewed as an internally produced, high-level cognitive representation of emotion. In addition, we may also observe enhanced activations in LIFG because of its role in combinatorial processing (Hagoort, 2005, 2013), as well as other languagerelevant regions in the temporal cortex, the ventromedial pFC, and/or the angular gyrus (Friederici, 2012; Poeppel, Emmorey, Hickok, \& Pylkkänen, 2012). Moreover, implied emotion or, more generally speaking, implied meaning can also be computed via inferential processing. Inferential processing is related to combinatorial processing as both compute meaning beyond the single word level. ${ }^{1}$ We view combinatorial processing as a necessity for language, whereas inferential processing is available when called upon by communicative needs, which is often the case during language communication. Several studies have shown that inferring the speaker meaning involves the theory of mind network including the TPJ and the mPFC (Bašnáková, Weber, Petersson, van Berkum, \& Hagoort, 2014; Hagoort 2013; van Ackeren, Casasanto, Bekkering, Hagoort, \&
Rueschemeyer, 2012; Kuperberg, Lakshmanan, Caplan, \& Holcomb, 2006).

\section{METHODS \\ Participants}

Twenty-four native Dutch speakers participated in the study for payment. Eight were excluded for the following reasons: Two did not fit the language criterion of being a native speaker of Dutch, two had noncorrected vision during the experiment, and four had too much movement during scanning $(>3.5 \mathrm{~mm})$. The remaining 16 participants $\left(14\right.$ women, ${ }^{2}$ mean age $=20.5$ years, range $=18$ 28 years) were right-handed, as assessed by the Dutch version of the Edinburgh handedness inventory (Oldfield, 1971). All had normal or corrected-to-normal vision, and none had a history of neurological, psychiatric, or language disorder problems. None was particularly depressed or happy, as assessed by the Positive Affect Negative Affect System Trait questionnaire (Watson, 1988; positive affect $=3.59, S D=0.34$, range $=3.1-4.0$, on a scale from 1 to 5 ; negative affect $=1.87, S D=0.44$, range $=1.3-2.7$ ). The study fell under the legal ethical approval procedure in the Netherlands. Before the experiment, each participant signed an informed consent and was told that they could stop their participation any time.

\section{Materials}

The materials consisted of 140 pairs of negative/neutral sentences (Table 1), 140 pairs of negative/neutral words (Table 1), and 140 pairs of negative/neutral scenes. We constructed the sentences from scratch and selected words based on the Dutch Affective Word norm database (Moors et al., 2013) and the word frequency database SubtLex-NL (Keuleers, Brysbaert, \& New, 2010). The semantic content of the words and sentences are matched, but not with the pictures. We selected pictures from the International Affective Picture System (Lang, Bradley, \& Cuthbert, 2008) and from the Internet because of the lack of animate beings in the International Affective Picture System neutral images. Several norming tests were carried out to verify the manipulated content and the quality of materials detailed below.

For verifying the emotional manipulations of all materials, 41 additional participants who did not take part in the scanning session participated in the computerized pretests for sentences (13 participants), words (13 participants), and pictures (15 participants). Participants rated each stimulus on their valence, arousal, and concreteness dimensions by clicking on a 9-point Self-Assessment Manikin scale, adapted from Bradley and Lang (1994), a rating scheme typically used in emotion research. The emotional scale ranged from a frowning manikin at the negative end $(=1)$ to a neutral-faced manikin in the middle $(=5)$ and a smiling manikin on the right $(=9)$. Likewise, the arousal 
Table 1. Examples of Negative/Neutral Sentences/Words in Dutch with English Translations Provided in Quotes

\begin{tabular}{|c|c|c|c|}
\hline Eg. & Condition & Sentences & Words \\
\hline \multirow[t]{4}{*}{1} & Negative & Ze kwam uit bet water en zag dat er een zwart beest vastgezogen zat aan haar been. & bloedzuiger \\
\hline & & "She came out of the water and saw a black animal attached on her leg." & "leech" \\
\hline & Neutral & Ze ging naar de keuken en zag dat er een paddenstoel klaargelegd was op bet aanrecht. & champignons \\
\hline & & "She went into the kitchen and saw some mushrooms laid on the counter." & "mushrooms" \\
\hline \multirow[t]{4}{*}{2} & Negative & De man zag een rood spoor op de vloer en zag zijn vrouw in de gang liggen. & bloedsporen \\
\hline & & "The man saw a red trace on the floor and saw his wife lying in the hallway." & "blood traces" \\
\hline & Neutral & De man bekeek de stadswandeling op de kaart en zag het oude gebouw op de boek liggen. & monumenten \\
\hline & & "The man checked the citywalk on the map and saw the old building lying at the corner." & "monuments" \\
\hline \multirow[t]{4}{*}{3} & Negative & Hij heeft nog maar één been en nu moet deze ook verwijderd worden. & amputatie \\
\hline & & "He has only one leg and now this one must also be removed." & "amputation" \\
\hline & Neutral & Hij heeft nog maar één bord en nu moeten er meer gekocht worden. & aardewerk \\
\hline & & "He has only one plate so now more must be purchased." & "pottery" \\
\hline \multirow[t]{4}{*}{4} & Negative & Er kropen honderden beestjes over haar benen die bun tanden in haar vlees zetten. & insectenbeet \\
\hline & & $\begin{array}{l}\text { "Hundreds of small animals were crawling on her legs and were putting their teeth into } \\
\text { her flesh." }\end{array}$ & "insect bite" \\
\hline & Neutral & Er reden honderden auto's over de weg waarvan de achterste lampen op bet wegdek schenen. & achterlichten \\
\hline & & "Hundreds of cars were driving on the road of which the rear lights were shining on the road" & "tail lights" \\
\hline
\end{tabular}

scale ranged from a nonarousing manikin on one end $(=1)$ to a more arousing manikin in the middle $(=5)$ and a very arousing manikin on the right $(=9)$. The concreteness scale ranged from concrete $(=1)$ to abstract $(=9)$.

The rating results are summarized in Table 2 . Overall, negative items are indeed more negatively valenced than neutral items for sentences, words, and pictures, with similar negative-neutral difference between sentences, words, and pictures. Paired $t$ tests indicated that, in sentences, the negative sentences were more negative than the neutral ones $(t(12)=19.796, p<.0001)$, more arousing than the neutral ones $(t(12)=5.022, p<.0001)$, and as concrete as the neutral ones $(t(12)=1.172, p=.264)$.
For words, the negative words were more negative than the neutral ones $(t(12)=12.041, p<.0001)$, more arousing than the neutral ones $(t(12)=7.321, p<.0001)$, and as concrete as the neutral ones $(t(12)=1.664, p=.122)$. In pictures, the negative scenes were more negative than the neutral ones $(t(14)=14.749, p<.0001)$, more arousing than the neutral ones $(t(14)=8.917, p<.0001)$, and as concrete as the neutral ones $(t(14)=1.050, p=.311)$.

Crucially, for sentences, we made sure that (1) the words in the negative and neutral sentences were matched in terms of the emotional characteristics and nonemotional features at the single word level, so that all emotional meaning came from combinations of words and not from

Table 2. Pretest Ratings of Valence, Arousal, and Concreteness for Negative and Neutral Sentences, Words, and Pictures on a 9-Point Scale

\begin{tabular}{|c|c|c|c|c|c|}
\hline & & $\begin{array}{c}\text { Sentences: Whole Sentence, } \\
\text { Mean (SD) }\end{array}$ & $\begin{array}{c}\text { Sentences: Content Words, } \\
\text { Mean (SD) }\end{array}$ & $\begin{array}{c}\text { Words, } \\
\text { Mean (SD) }\end{array}$ & $\begin{array}{l}\text { Pictures, } \\
\text { Mean (SD) }\end{array}$ \\
\hline \multirow[t]{2}{*}{ Valence } & Negative & $2.36(0.5)$ & $4.40(0.32)$ & $2.38(0.5)$ & $2.38(0.6)$ \\
\hline & Neutral & $5.22(0.2)$ & $4.47(0.36)$ & $5.48(0.6)$ & $5.16(0.4)$ \\
\hline \multirow[t]{2}{*}{ Arousal } & Negative & $5.59(1.1)$ & $4.16(0.36)$ & $5.62(1.1)$ & $5.90(1.8)$ \\
\hline & Neutral & $3.11(1.5)$ & $4.18(0.45)$ & $2.71(1.4)$ & $1.95(0.9)$ \\
\hline \multirow[t]{2}{*}{ Concreteness } & Negative & $6.41(1.1)$ & - & $6.37(1.2)$ & $6.58(1.1)$ \\
\hline & Neutral & $6.20(1.3)$ & - & $6.72(1.3)$ & $6.79(1.2)$ \\
\hline
\end{tabular}


words themselves; (2) the negative and neutral sentences were matched in terms of their nonemotional implied meanings, so that the inferential processing was matched between conditions; and (3) the negative and neutral sentences differed in terms of their emotional implied meanings.

Regarding (1), we matched the mean valence, arousal, and word frequencies of the content words of the sentences between conditions. We ensured this by first finding the valence and arousal values of all of the content words in the sentences based on the Dutch Affective Word norm database (Moors et al., 2013), averaging the values of the content words of a given sentence and matching the averages between the conditions. In this norm, the rating scale ranges from 1 to $7(1=$ very negative, $7=$ very positive; $1=$ very calm and $7=$ very arousing). The averaged valence ratings were $4.4(S D=0.3$, range $=3.5-4.9)$ for the content words in the negative sentences and $4.5(S D=$ 0.4 , range $=3.4-5.8)$ in the neutral sentences $(p=.998)$. The averaged arousal ratings were $4.2(S D=0.4$, range $=$ 3.2-4.8) for the content words in the negative sentences and $4.2(S D=0.5$, range $=3.1-5.1)$ for the content words in the neutral sentences $(p=.926)$. We consulted Keuleers et al. (2010) for word frequency values. The averaged log frequencies of the content words were $2.3(S D=0.4)$ for the negative sentences and $2.2(S D=0.4)$ for the neutral sentences $(p=.389)$.

Regarding (2), the nonemotional implied meaning and animacy were assessed by two native Dutch-speaking research assistants. One of the assistants first wrote down for each sentence what the nonemotional implied meaning was. For example, for a sentence such as "The window was open and the paper on the desk fell to the floor," the research assistant wrote down "There was wind," as the nonemotional implied meaning. The other then rated whether the generated nonemotional implied meaning was emotional $(=1)$ or nonemotional $(=0)$. The averaged ratings were $0.02(S D=0.15)$ for the negative and 0.08 $(S D=0.3)$ for the neutral sentences, with the difference being nonsignificant $(p=.09) .{ }^{3}$ For animacy, both assistants judged whether there was anything animate in a given sentence $(1=y e s, 0=n o)$. The averaged scores for animacy were $1.0(S D=0.2)$ for the negative and 0.9 $(S D=0.2)$ for the neutral sentences $(p=.741)$. The assistants also discussed and made sure that the syntactic structures are matched. The sentence length was measured both in terms of the number of letters and words. The averaged numbers of letters were $78.4(S D=15.2)$ for the negative and $80.6(S D=17.4)$ for the neutral sentences $(p=.424)$, and the averaged numbers of words were 14.9 $(S D=3.1)$ for the negative and $14.7(S D=3.0)$ for the neutral sentences.

Regarding (3), two research assistants generated one sentence that they considered to represent the implied meaning for each sentence. Then, 15 participants rated whether those generated sentences were negative on a $1-7$ scale $(1=$ negative, $4=$ neutral, $7=$ positive $)$.
On average, implied meanings generated from the negative sentences were rated $1.80(S D=0.31)$ and implied meanings generated from the neutral sentences were rated $4.53(S D=0.59)$. This difference was statistically significant $(p<.0001)$, confirming our manipulation.

Additionally, 20 nonsense sentences (e.g., The patch will gladly reveal against a watery involved magic.), 20 nonwords, and 20 pictures with a black dot somewhere in the picture were created as the catch trials.

\section{Procedure}

Participants were debriefed and signed an informed consent form before entering the scanner room and lying down in the scanner. Each participant did three runs (Figure 1), with the anatomical scan and breaks in between runs. In Run 1, 70 negative sentences, 70 neutral sentences, and 20 nonsense sentences were presented in randomized order via the Presentation software (Neurobehavioral Systems, Inc., www.nbs.com). In each trial, a fixation sign "+" was presented for $500 \mathrm{msec}$ to signal the upcoming sentence. The presentation duration of a given sentence was length dependent (the number of words $\times 500 \mathrm{msec}$ ) if the number of words in a sentence was $\leq 8$. If the number of words was $>8$, the sentence was presented for $4000 \mathrm{msec}$. Between trials, a black screen was presented with the intertrial interval varying from $2.5 \mathrm{sec}$ to $5.5 \mathrm{sec}$ (mean $=4 \mathrm{sec}$ within a participant) to effectively jitter trial onset with respect to volume acquisition (Dale, 1999).

In Run 2, 70 negative words, 70 neutral words, and 20 nonwords were presented in random order. The trial structure was the same as Run 1 except for the presentation duration of the stimulus word. If the word length was $\leq 8$ letters, the word was presented for (the number of letters $\times 50)$ msec. If the length was $>8$ letters, the word was presented for 1500 msec. In Run 3, 70 negative pictures, 70 neutral pictures, 10 negative pictures with a black dot, and 10 neutral pictures with a black dot were presented in random order. The trial structure was the same as Runs 1 and 2, except for the presentation duration of the stimulus picture $(2.5 \mathrm{sec})$.

Participants were instructed to read/view the sentences/ words/pictures attentively and only press a button when the sentence did not make sense, when the word was not a real word, and when a black dot appeared somewhere in the picture. Participants had four practice trials before the run, with materials not used in the actual experiment. After the scanning, a short exit interview was conducted to verify if the experiment went well, for example, double-checking if the participants could see the sentences well.

\section{fMRI Data Acquisition}

EPIs covering the whole brain were acquired with an eightchannel head coil on a Siemens MR system with a magnetic 
Figure 1. Illustration of the negative sentences, words, and pictures in each of the runs.

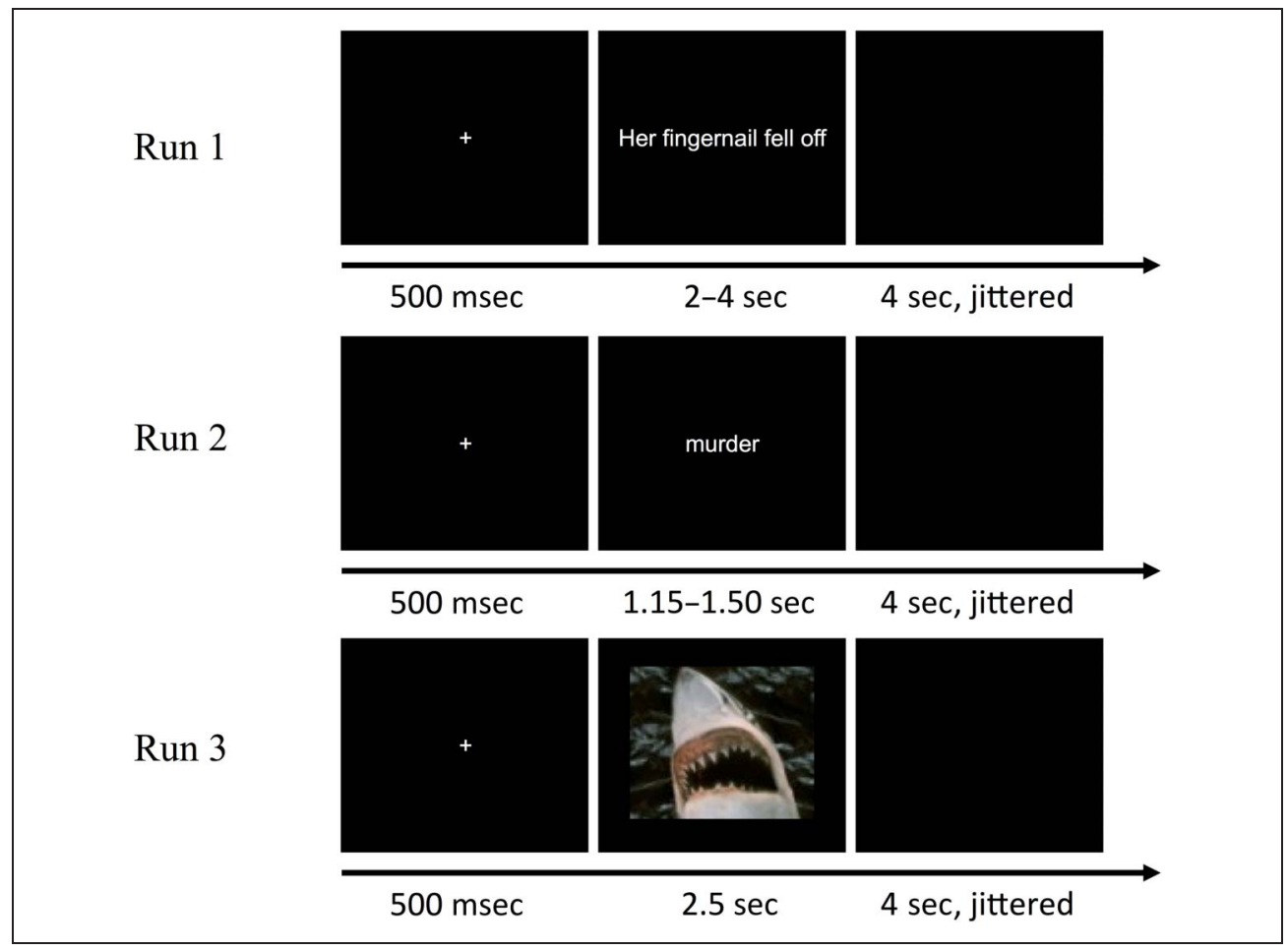

field strength of $1.5 \mathrm{~T}$ (repetition time $=2340 \mathrm{msec}$; echo time $=35$ msec; flip angle $=90^{\circ}$ ). Each volume consisted of 32 transversal slices. The voxel size was $3.3 \mathrm{~mm} \times$ $3.3 \mathrm{~mm} \times 3.5 \mathrm{~mm}$. Additionally, a high-resolution T1weighted anatomical scan of the head was taken (repetition time $=2250 \mathrm{msec}$; echo time $=2.95 \mathrm{msec} ; 1 \mathrm{~mm}$ isocubic voxel size).

\section{fMRI Data Analysis}

Data were analyzed with Statistical Parametric Mapping 8 (www.fil.ion.ucl.ac.uk/spm/software/spm8/). Preprocessing involved realignment through rigid body registration to correct for head motion and correction for differences in slice timing acquisition to the onset of the first slice. Subsequently, for each participant the anatomical scan was normalized to Montreal Neurological Institute (MNI) space, and normalization parameters were applied to all functional (EPI) images. Functional data were interpolated to $2 \mathrm{~mm} \times 2 \mathrm{~mm} \times 2 \mathrm{~mm}$ voxel size and spatially smoothed with an isotropic Gaussian kernel of $8 \mathrm{~mm}$ FWHM. First-level analysis involved estimation of beta weights in a multiple regression analysis with regressors describing the expected hemodynamic responses (Friston et al., 1995) for each of the conditions (Sentence_negative, sentence_neutral, word_negative, word_neutral, picture negative, picture_neutral). Stimuli were modeled as their actual duration, and regressors were convolved with a canonical two gamma hemodynamic response function (e.g., Friston, Holmes, Poline, Price, \& Frith, 1996). The six motion parameters obtained from the motion correction algorithm (three translations and three rotations) were included as regressors of no interest. Second-level group analysis involved testing a mixed model with participants as a random factor ("random effects analysis"; Friston, Holmes, Price, Buchel, \& Worsley, 1999). Group statistical maps were corrected for multiple comparisons by combining an activation level threshold of $p<.001$ at the individual participant level with a cluster extent threshold computed using the theory of Gaussian random fields to arrive at a statistical threshold with a $p<.05$ significance level, corrected for multiple comparisons (Poline, Worsley, Evans, \& Friston, 1997; Friston et al., 1996).

\section{ROI Analysis}

To understand implied emotion and how it may be related to combinatorial processing and/or motor simulation, we assessed responses from the IFG including its three subdivisions, pars opercularis, pars triangularis, and pars orbitalis, and motor regions including motor cortex and premotor cortex (Eickhoff et al., 2005). We created these ROIs by using the automated anatomical labeling template based on a landmark-guided parcellation of the MNI template (Tzourio-Mazoyer et al., 2002).

We additionally carried out ROI analyses for typical emotion regions, including amygdala, $\mathrm{mPFC}$, and insula. The ROIs for amygdala and mPFC were anatomically defined. As for the ROI for the anterior insula, because the insula comprises a very large part of the cortex and different subparts of the insula are known to be differently involved in cognitive processing, this ROI was created on the basis of an extensive meta-analysis of neuroimaging studies investigating emotional processing (Kober et al., 2008). The 
mean coordinates of the maxima described in the metaanalysis were taken for the left and right insula separately (Kober et al., 2008; Table 3). The left insula coordinates $[x y z]$ are $\left[\begin{array}{lll}-28 & 6 & -22\end{array}\right],\left[\begin{array}{ll}-40 & 24-6\end{array}\right],[-3412-10],[-40$ $10-20]$, and the right insula coordinates are [42 $24-8$ ], [40 4 - 14 ], [ $4416-2$ ]. The ROIs were spheres with the mean coordinates for each hemisphere as center ( $\mathrm{L}:-36$ $13-15$, R: $4215-8$ ), with a $10-\mathrm{mm}$ radius.

\section{RESULTS}

\section{Behavioral Results}

The accuracies for the catch trials were $99.1 \%(S D=2.0 \%)$ for sentences in Run 1, 100\% (SD = 0) for words in Run 2, and $99.1 \%(S D=3.8 \%)$ for pictures in Run 3 .

\section{Whole-brain Analysis Results}

\section{Sentences}

Comparing negative versus neutral sentences revealed increased activation in a large cluster stretching from the left insular cortex to the hippocampus including the amygdala (Figure 2; Table 3). A similar but less extended cluster of activation was also observed in the right hemisphere. Bilateral activation of the middle temporal gyrus, the
Table 3. Whole-brain (Corrected): Negative versus Neutral Sentences (Significance Level $p<.05$, Corrected)

\begin{tabular}{lrrrl}
\hline Sentences & $x$ & $y$ & $z$ & $T$ \\
\hline Negative $>$ Neutral & & & & \\
R Insula & 26 & 10 & -10 & 5.49 \\
& 28 & 12 & -22 & 3.2 \\
LR medial superior frontal gyrus & -2 & 38 & 42 & 7.12 \\
& -2 & 54 & 30 & 7.22 \\
& -14 & 46 & 24 & 6.51 \\
LR amygdala & -30 & 2 & -22 & 6.32 \\
& 24 & -12 & -8 & 5.51 \\
L insula & -30 & 18 & -4 & 3.6 \\
LR middle temporal gyrus & -54 & -30 & -8 & 4.91 \\
& 56 & -36 & 0 & 3.85 \\
R inferior frontal gyrus & 54 & 26 & -2 & 5.66 \\
L precuneus & -8 & -60 & 30 & 5.26
\end{tabular}

Neutral > Negative

$\mathrm{R}$ superior frontal sulcus

28

2

$62 \quad 4.64$
Figure 2. Activations for the negative $>$ neutral contrast in sentences (yellow), words (red), and pictures (blue) and their overlap (green). All activations are corrected for multiple comparisons as described in the Methods section $(p<.05)$.

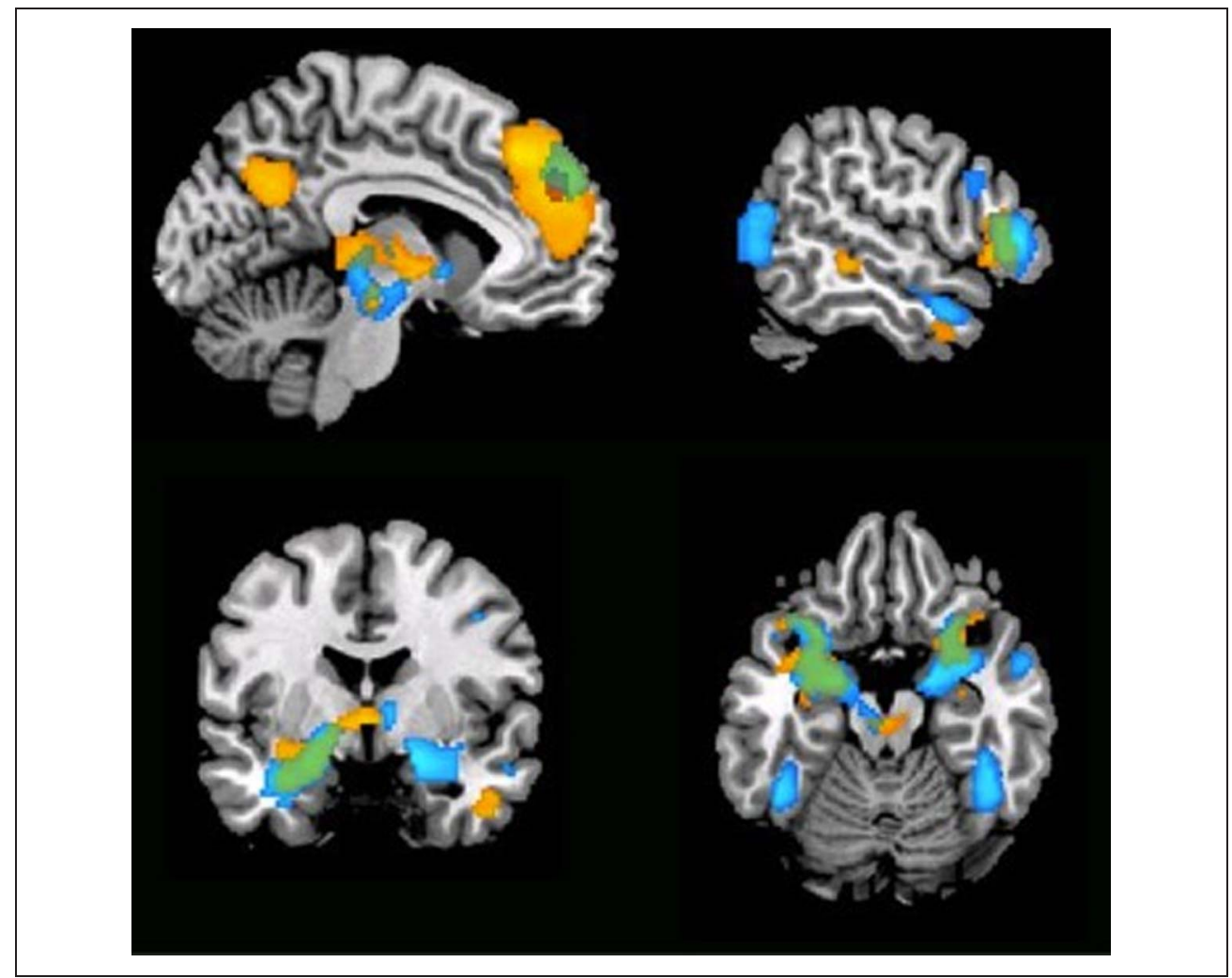


medial superior frontal gyrus (mPFC), the left precuneus, and the right inferior frontal gyrus (RIFG) were observed. The opposite contrast (neutral $>$ negative) only revealed one cluster of activation in the right superior frontal sulcus.

\section{Words}

Comparing negative versus neutral words revealed one cluster of activation in the left medial superior frontal gyrus (mPFC; Figure 2; Table 4). The opposite contrast (neutral $>$ negative) did not reveal any activation.

\section{Pictures}

Comparing negative versus neutral pictures led to widespread bilateral activation in the limbic system, including the amygdalae, stretching into the temporal poles (Figure 2; Table 5). Another cluster of activation was found in the bilateral fusiform gyrus, stretching into the inferior and posterior middle temporal cortices. Additionally, clusters of activation were observed in both LIFG and RIFG, with the cluster on the right being much larger than that on the left. The opposite contrast (neutral > negative) showed a set of brain regions some of which have been implicated as part of the default mode network, including the bilateral superior parietal cortex, bilateral posterior insula, posterior cingulated cortex, and bilateral anterior middle frontal sulci.

\section{Conjunction Analysis Results}

We next performed a conjunction analysis, testing for regions involved in negative $>$ neutral in all three modalities (i.e., Sentence negative $>$ Sentence neutral $\cap$ Picture negative $>$ Picture neutral $\cap$ Word negative $>$ Word neutral). We tested for the so-called conjunction null (Nichols, Brett, Andersson, Wager, \& Poline, 2005), in which each statistical map is thresholded at $p<.05$ corrected, using the correction procedure described above. This revealed one area of activation in the medial superior frontal gyrus (mPFC; MNI peak voxel -6 49 32; Figure 3).

Table 4. Whole-brain (Corrected): Negative versus Neutral Words (Significance Level $p<.05$, Corrected)

\begin{tabular}{lcccc}
\hline Words & $x$ & $y$ & $z$ & $T$ \\
\hline Negative $>$ Neutral & & & & \\
L medial superior frontal gyrus & -8 & 50 & 32 & 4.47 \\
& & & & \\
Neutral $>$ Negative & & & & \\
- & - & - & - & - \\
\hline
\end{tabular}

Table 5. Whole-brain (Corrected): Negative versus Neutral Pictures (Significance Level $p<.05$, Corrected)

\begin{tabular}{lrrrl}
\hline Pictures & $x$ & $y$ & $z$ & $T$ \\
\hline Negative $>$ Neutral & & & & \\
LR amydalae, stretching into the & -22 & -6 & -16 & 6 \\
$\quad-35$ & 0 & -28 & 3.8 \\
$\quad$ temporal poles & 20 & -4 & -16 & 7.14 \\
& 44 & 16 & -32 & 5.87 \\
& 52 & 28 & 0 & 4.88 \\
LR inferior frontal gyrus & 50 & 14 & 28 & 4.35 \\
& -42 & 28 & 8 & 3.92 \\
LR fusiform gyrus & -44 & -48 & -16 & 6.58 \\
& 44 & -44 & -14 & 7.11 \\
LR inferior temporal sulcus & -42 & -74 & -4 & 4.64 \\
& 40 & -72 & -10 & 5.64 \\
LR posterior middle temporal & 46 & -68 & 8 & 5.1 \\
gyrus & -44 & -70 & 8 & 4.59 \\
L medial superior frontal gyrus & -4 & 56 & 32 & 6.3
\end{tabular}

Neutral $>$ Negative

LR posterior insula

$$
\begin{array}{rrrr}
-43 & -21 & 12 & 4.5 \\
50 & -56 & 46 & 8.3
\end{array}
$$$$
\begin{array}{llllll}
\text { LR superior parietal sulcus } & -48 & -56 & 46 & 6.55
\end{array}
$$$$
\begin{array}{lllll} 
& 50 & -54 & 46 & 9.21 \\
\text { Posterior cingulated cortex } & -4 & -77 & 46 & 5.35
\end{array}
$$$$
\begin{array}{lllll}
\text { LR middle frontal sulcus } & 30 & 56 & 6 & 6.57
\end{array}
$$$$
\begin{array}{llll}
-30 & 50 & 8 & 5.69
\end{array}
$$

\section{ROI Analysis Results}

\section{Inferior Frontal Cortex}

We examined inferior frontal cortex and its subdivisions of pars opercularis, pars triangularis, and pars orbitalis, bilaterally, for sentences and for words. The results are summarized in Figure 4. Mean activation level indicated by beta weights was extracted and entered into a repeatedmeasures ANOVA of 2 Conditions (negative, neutral) $\times$ 2 Hemispheres (left, right) $\times 3$ Locations (pars opercularis, pars triangularis, pars orbitalis). In sentences, there was a Condition $\times$ Location interaction $[F(2,30)=5.001, p=$ .026] with no hemisphere interaction $(F<1)$. Negative sentences showed more activation than neutral sentences in pars orbitalis $[F(1,15)=16.413, p=.001]$ and pars triangularis $[F(1,15)=9.445, p=.008]$, but not in pars 


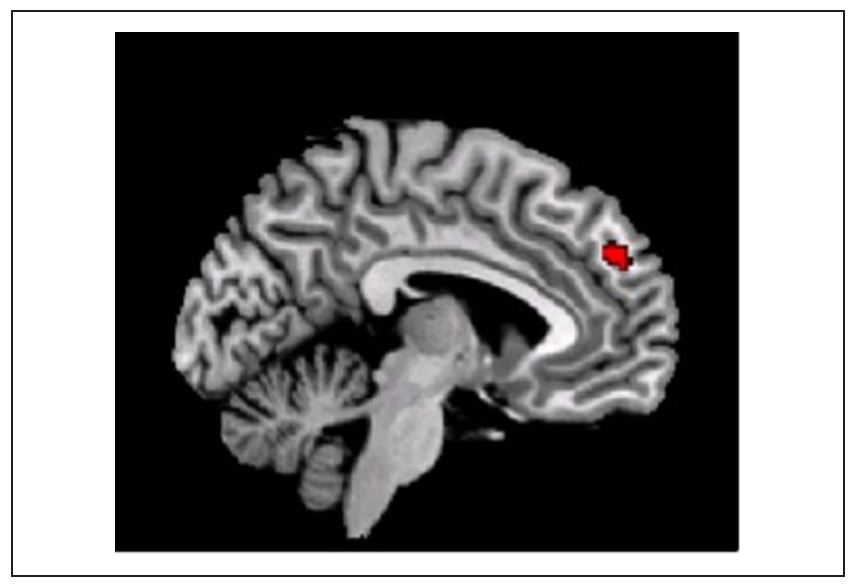

Figure 3. Conjunction analysis of the negative $>$ neutral contrast in sentences, words, and pictures. All activations are corrected for multiple comparisons as described in the Methods section $(p<.05)$.

opercularis $[F(1,15)=1.87, p=.192]$. For words, there was no significant effect.

\section{Motor Areas}

We examined motor areas including motor cortex (BA 4) and premotor cortex (BA 6). Repeated-measures ANOVAs of 2 Condition $\times 2$ Hemisphere revealed no Condition $\times$ Hemisphere interaction or any main effect of condition, neither in motor $(F<1)$ nor in premotor areas $(F<1)$, for both sentences and words.

\section{Emotion Network}

We carried out focused analyses for sentences and words in the following ROIs: amygdala, mPFC, and insula. The results are summarized in Figure 5. In amygdala, for sentences, a repeated-measures ANOVA of 2 Condition $\times$ 2 Hemisphere revealed a main effect of condition $[F(1$, $15)=16.12, p<.001]$. The amygdala was more activated for negative than neutral sentences in both hemispheres [left: $F(1,15)=18.45, p<.001$; right: $F(1$, $15)=8.37, p<.01]$. For words, there was no significant effect.

In $\mathrm{mPFC}$ (Figure 5, middle) for sentences, there was a Condition $\times$ Hemisphere interaction $[F(1,15)=17.73$, $p=.001]$. Negative sentences led to more activations than neutral sentences in both hemispheres [left: $F(1$, $15)=67.55, p<.0001$; right: $F(1,15)=33.31, p<$ $.0001]$, and such difference was greater in the right than in the left hemisphere. For words, there was also a Condition $\times$ Hemisphere interaction $[F(1,15)=10.42$, $p=.006]$. The negative $>$ neutral difference in the right mPFC was larger than that in the left. Within each hemisphere, there was, however, no main effect of condition [left: $F(1,15)=1.55, p=.23$; right: $F<1$ ].

For the insula (Figure 5, bottom), we obtained a Condition $\times$ Hemisphere interaction $[F(1,15)=6.45$, $p=.02]$. Pairwise comparisons indicate that the activation level was higher for negative than for neutral sentences, but only in the left hemisphere $[F(1,15)=$ $14.71, p=.002]$. For words, no significant effect was found.
Figure 4. Mean activations for neutral (dark gray bars) and negative (light gray bars) sentences and words for IFC pars orbitalis (top), IFC pars triangularis (middle), and IFC pars orbitalis (bottom) in the left hemisphere (left column) and the right hemisphere (right column).

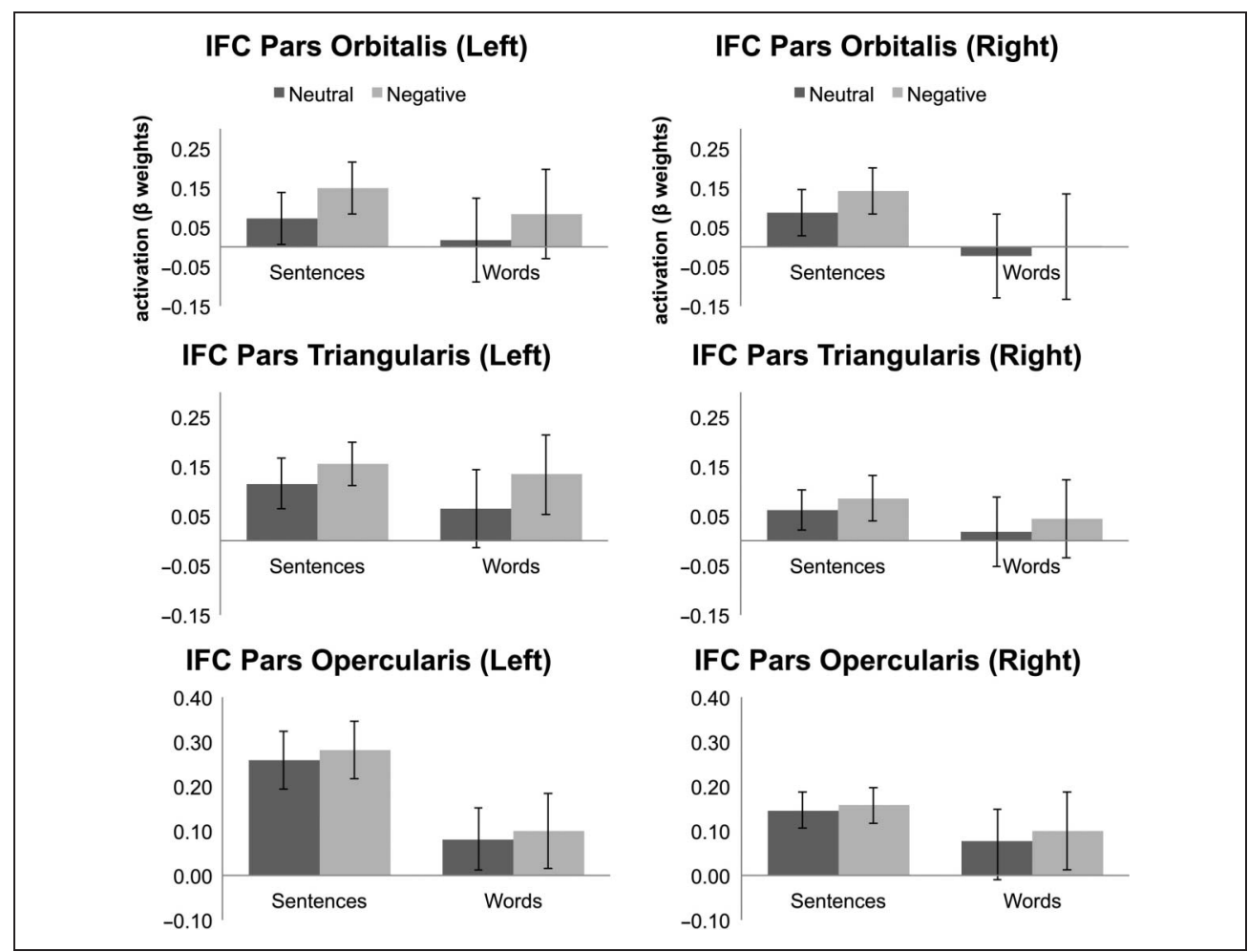


Figure 5. Mean activations for neutral (dark gray bars) and negative (light gray bars) sentences and words for amygdala (top), medial prefrontal cortex (middle), and insula (bottom) in the left hemisphere (left column) and the right hemisphere (right column).

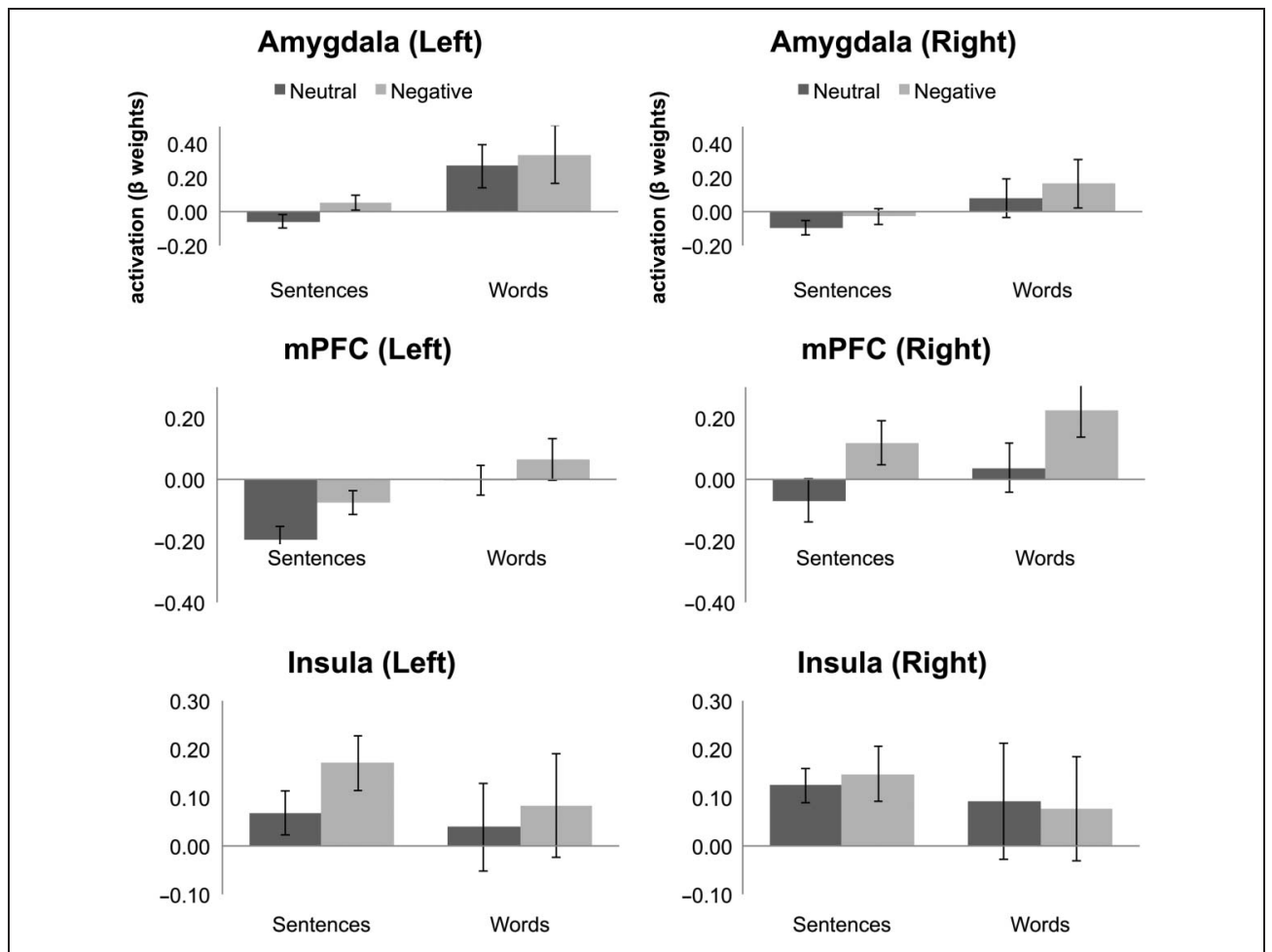

\section{DISCUSSION}

This study is one of the first studies that investigated the brain regions for the comprehension of implied emotion in sentences. Participants read negative sentences without negative words, for example, "The boy fell asleep and never woke up again," and their neutral counterparts "The boy stood up and grabbed his bag." This kind of negative sentence allows us to examine implied emotion derived at the sentence level, without associative emotion coming from word retrieval. We found that implied emotion led to activations in emotion-related areas including the amygdala, insula, and mPFC. In addition, we found that implied emotion also led to increased activation in language-related areas including the IFG. No activation in the motor areas was observed.

\section{Regions for Implied Emotion in Sentences}

The most pronounced activation in sentences is within the mPFC, which is also the activation overlap across the three stimulus formats examined in the current study. There are at least three functional interpretations. First, the mPFC has been implicated in general emotion processing (Lane et al., 1997). The finding that all three stimulus formats have led to activations in the $\mathrm{MPFC}$ is consistent with this proposal. Adding to this literature, we showed that the $\mathrm{mPFC}$ is involved not only in associative emotion in words and pictures but also in implied emotion in sentences. Within the MPFC, the activation for the implied emotion in our negative sentences was more dorsal than ventral.
This is in line with past studies reporting larger signal changes in the dorsal than the ventral mPFC when participants interpreted the same stimuli (faces) as being negatively valenced as opposed to being positively valenced (Kim, Somerville, Johnstone, Alexander, \& Whalen, 2003).

Second, the MPFC has been implicated in the cognitive aspect of emotion processing. Implied emotion in sentences requires combinations of words and therefore is likely to be more cognitively costly than retrieving emotion explicitly associated with words, as reflected by the greater mPFC activation in sentences than in words. With regard to the subdivision of the mPFC for mentalizing, it has been shown that the ventral $\mathrm{mPFC}$ is more involved in the evaluative judgments about self, whereas the dorsal mPFC is more involved in mentalizing about others (Denny, Kober, Wager, \& Ochsner, 2012). This fits our observation of the dorsal mPFC activation in sentences, as our sentences contain no first person pronouns and are always about the sufferings of others. Future studies can manipulate the first/third person pronouns in sentences with implied emotion to further verify this interpretation.

Third, a number of language studies, the MPFC has been implicated in inferential processes such as causal inferencing (Ferstl, 2010; Kuperberg et al., 2006; Ferstl \& von Cramon, 2002), indirect speech (van Ackeren et al., 2012), and conversational implicatures (Bašnáková et al., 2014). Thus, the observed activation in the MPFC could be driven by general inferential processing. This interpretation would suggest that more inferential processing was needed for the condition with implied meaning than the one without, rendering the output of the inferential 
processing (emotion, in this case) not crucial as a function of the mPFC. Although this is possible, it is unlikely, as the $\mathrm{mPFC}$ is found in the vast majority of emotion studies. In addition, in the current study, the amount of nonemotional inferential processing was matched between conditions (cf. Materials). A more likely scenario is that the implied meaning was jointly output via inferential processing in $\mathrm{mPFC}$ in addition to the core language combinatorial processing in areas such as LIFG. After all, successful communication requires both coded meaning and speaker meaning.

A second prominent activation is found in the insula for implied emotion in sentences, but not in words or pictures. The insula is important for social emotion such as compassion and empathy (Lamm \& Singer, 2010). The insula activation we observed may reflect our readers' compassionate/empathizing feelings about the scenarios described in the sentences. This latter interpretation is consistent with Willems et al. (2011), who found activation in the left anterior insula for fearful relative to neutral captions without scenes. They suggested that their participants "feel along" with the implied meaning of the fearful captions and that the insula is involved when explicit attention is drawn to emotion (Singer, Critchley, \& Preuschoff, 2009).

A third prominent activation for implied emotion in sentences is found in the amygdala. This is not surprising given that our negative sentences were rated more arousing than the neutral ones and that past research has shown that amygdala responds to the arousing dimension of the stimuli (cf. Adolphs et al., 1999). An interesting next question is whether implied emotion could be more arousing than sentences that convey emotion explicitly. This is an empirical question, but we suspect that this is probably not necessarily the case, at least not based on comparing the present data with the one study that found amygdalar responses for sentences with explicit emotion (Moll et al., 2002; Holt et al., 2011).

\section{Regions for Associative Emotion in Words and Pictures}

For words, the only area more activated for the negative relative to neutral words is the $\mathrm{mPFC}$, which has been shown before in a number of studies on emotional words (Herbert et al., 2009; Cato et al., 2004; Tabert et al., 2001). Relating to the mentalizing function in the mPFC, our results indicate that reading negative words requires more cognitive processing of emotion than neutral words. However, no activation in amygdala is observed, although the valence/arousal difference between the negative and neutral words has been matched to be the same as that in the sentences (Table 2). The lack of amygdala activation may be due to the nonevaluative nature of our passive reading task, which would be consistent with the literature that so far only studies that employed an evaluative task on words found amygdalar responses (cf. Introduction). However, this cannot account for the amygdala activation that we observed for the emotional sentences, because they were read passively as well. We contend that emotional language can lead to activation in amygdala, but only when there is sufficient context including both the speaker context (task/processing goal) and the sentential context that might make the emotional information salient and noticeable.

In pictures, negative pictures led to more activation in the bilateral amygdala and $\mathrm{mPFC}$ relative to neutral words, findings that are typically observed in emotion research. There was also enhanced activation in IFG and middle temporal gyrus (MTG). It is not uncommon to observe activation in IFG in imaging studies of emotional scenes (Vytal \& Hamann, 2010). The IFG and MTG are also commonly implicated in language studies. It is possible that our participants made up stories for the scenarios depicted in the pictures internally, leading to some verbal processing. The latter explanation is supported by the ROI analyses, which showed that both pars triangularis and pars orbitalis are involved, which are usually regarded as nodes in the language processing network (Hagoort, 2005, 2013). It should be noted that the latter interpretation was constructed post hoc, because we had no a priori expectation that participants would engage in more verbalization in the negative as compared to the neutral condition.

\section{Regions for Combinatorial Processing in Sentences}

Implied emotion in sentences enhanced activation in the RIFG and bilateral MTG in the whole-brain analysis and bilateral IFG in the ROI analysis. These areas are known to be critical for language processing, with an especially important contribution of left and right IFG to combinatorial operations in language. Through these operations, sentence level meaning is constructed from the meanings of the individual lexical items. Crucially, in our sentence condition, the emotional content was not due to the meaning of the individual words but resulted fully from the combinatorial operations that created the overall sentence meaning, with, in our case, an emotional quality.

The coactivation of these language-relevant areas suggests that the emotion network is intricately connected with the processing in the left and right IFG. As concerns right IFG, previous studies have found that increased combinatorial processing leads to increased activation in the right IFG, especially for stimuli consisting of brief discourse (Ferstl, 2010; Hagoort, Baggio, \& Willems, 2009; Menenti, Petersson, Scheeringa, \& Hagoort, 2009). Within the subdivisions of the left IFG, enhanced activations in pars orbitalis and pars triangularis were found, but not in pars opercularis. Thus, implied emotion in visual sentences recruit the pars orbitalis and pars triangularis, most likely for the semantic and the syntactic processing that they support, respectively (Hagoort, 
2005). The left IFG and other language areas might be gain-modulated by the emotional content of the sentences as a result of its relevance for the organism as a whole. That is, information from the emotion network feeds back into the language network to increase the level of lexical activations and combinatorial operations, given the relevance of the emotional content.

Activations in some unpredicted regions were found. First, the negative $>$ neutral contrast in sentences led to activation in the left precuneus. The precuneus has been implicated in self-centered imagery and episodic memory retrieval (Cavanna \& Trimble, 2006). Perhaps the participants imagined themselves in the described situation more in the negative than in the neutral conditions. Second, the neutral $>$ negative contrast in sentences led to activation in the right superior frontal sulcus. The right superior frontal sulcus has been implicated in the maintenance of information (Taylor et al., 2004). It could be that the neutral sentences require more working memory for information maintenance than negative sentences because of the lack of emotional significance in the neutral sentences. Given that we did not have a priori hypotheses about these unexpected findings, our interpretations here remain speculations.

Finally, the present findings have implications for embodied theories of emotion. We did not find any involvement of the motor regions, which suggests that not all types of emotional meaning must rely on simulation in the motor regions and that implied emotion can be the result of combinatorial operations, a result that cannot be explained in terms of a Hebbian cell assembly of neurons coding for the linguistic elements and their emotional valence.

Reprint requests should be sent to Vicky Tzuyin Lai, Department of Psychology, University of South Carolina, 1512 Pendleton Street, Columbia, SC 29208, or via e-mail: vicky.tzuyin.lai@ gmail.com.

\section{Notes}

1. It is possible to dissociate combinatorial processing and inferential processing. It is possible to combine words to generate a new sentence without generating an inference, and it is possible to generate an inference without combining words to generate a new sentence.

2. Although we did not intend to selectively test female participants, after excluding bad participants the majority is female. It is possible that gender plays a role in the processing of implied emotion, which is an interesting issue for future investigation.

3. See Kuperberg et al. (2006) for another way of verifying inferences.

\section{REFERENCES}

Adolphs, R., Russell, J. A., \& Tranel, D. (1999). A role for the human amygdala in recognizing emotional arousal from unpleasant stimuli. Psychological Science, 10, 167-171.
Amodio, D. M., \& Frith, C. D. (2006). Meeting of minds: The medial frontal cortex and social cognition. Nature Reviews Neuroscience, 7, 268-277.

Bašnáková, J., Weber, K., Petersson, K. M., van Berkum, J., \& Hagoort, P. (2014). Beyond the language given: The neural correlates of inferring speaker meaning. Cerebral Cortex (New York, N.Y.: 1991), 24, 2572-2578.

Bemis, D. K., \& Pylkkänen, L. (2011). Simple composition: An MEG investigation into the comprehension of minimal linguistic phrases. Journal of Neuroscience, 31, 2801-2814.

Bradley, M. M., \& Lang, P. J. (1994). Measuring emotion: The self-assessment manikin and the semantic differential. Journal of Behavior Therapy and Experimental Psychiatry, 25, 49-59.

Brennan, J., \& Pylkkänen, L. (2012). The time-course and spatial distribution of brain activity associated with sentence processing. Neuroimage, 60, 1139-1148.

Cato, M. A., Crosson, B., Gökçay, D., Soltysik, D., Wierenga, C., Gopinath, K., et al. (2004). Processing words with emotional connotation: An fMRI study of time course and laterality in rostral frontal and retrosplenial cortices. Journal of Cognitive Neuroscience, 16, 167-177.

Cavanna, A. E., \& Trimble, M. R. (2006). The precuneus: A review of its functional anatomy and behavioural correlates. Brain: A Journal of Neurology, 129, 564-583.

Chang, L. J., Yarkoni, T., Khaw, M. W., \& Sanfey, A. G. (2013). Decoding the role of the insula in human cognition: Functional parcellation and large-scale reverse inference. Cerebral Cortex, 23, 739-749.

Dale, A. M. (1999). Optimal experimental design for event-related fMRI. Human Brain Mapping, 8, 109-114.

Damasio, A. R., Everitt, B. J., \& Bishop, D. (1996). The somatic marker hypothesis and the possible functions of the prefrontal cortex [and discussion]. Philosophical Transactions of the Royal Society of London, Series B, Biological Sciences, 351, 1413-1420.

Davis, M., \& Whalen, P. J. (2001). The amygdala: Vigilance and emotion. Molecular Psychiatry, 6, 13-34.

Denny, B. T., Kober, H., Wager, T. D., \& Ochsner, K. N. (2012). A meta-analysis of functional neuroimaging studies of self- and other judgments reveals a spatial gradient for mentalizing in medial prefrontal cortex. Journal of Cognitive Neuroscience, 24, 1742-1752.

Dunn, B. D., Dalgleish, T., \& Lawrence, A. D. (2006). The somatic marker hypothesis: A critical evaluation. Neuroscience \& Biobehavioral Reviews, 30, 239-271.

Eickhoff, S. B., Stephan, K. E., Mohlberg, H., Grefkes, C., Fink, G. R., Amunts, K., et al. (2005). A new SPM toolbox for combining probabilistic cytoarchitectonic maps and functional imaging data. Neuroimage, 25, 1325-1335.

Etkin, A., Egner, T., \& Kalisch, R. (2011). Emotional processing in anterior cingulate and medial prefrontal cortex. Trends in Cognitive Sciences, 15, 85-93.

Ferstl, E. C. (2010). Neuroimaging of text comprehension: Where are we now? Italian Journal of Linguistics, 22, 61-88.

Ferstl, E. C., \& von Cramon, D. Y. (2002). What does the frontomedian cortex contribute to language processing: Coherence or theory of mind? Neuroimage, 17, 1599-1612.

Friederici, A. D. (2012). The cortical language circuit: From auditory perception to sentence comprehension. Trends in Cognitive Sciences, 16, 262-268.

Friston, K. J., Holmes, A., Poline, J. B., Price, C. J., \& Frith, C. D. (1996). Detecting activations in PET and fMRI: Levels of inference and power. Neuroimage, 4, 223-235.

Friston, K. J., Holmes, A., Worsley, K. J., Poline, J.-B., Frith, C. D., \& Frackowiak, R. S. (1995). Statistical parametric maps in functional imaging: A general linear approach. Human Brain Mapping, 2, 189-210. 
Friston, K. J., Holmes, A. P., Price, C. J., Buchel, C., \& Worsley, K. J. (1999). Multisubject fMRI studies and conjunction analyses. Neuroimage, 10, 385-396.

Frith, C. D., \& Frith, U. (2006). How we predict what other people are going to do. Brain Research, 1079, 36-46.

Glenberg, A. M., Havas, D., Becker, R., \& Rinck, M. (2005). Grounding language in bodily states: The case for emotion. In The grounding of cognition: The role of perception and action in memory, language, and thinking (pp. 115-128). Cambridge: Cambridge University Press.

Graves, W. W., Binder, J. R., Desai, R. H., Conant, L. L., \& Seidenberg, M. S. (2010). Neural correlates of implicit and explicit combinatorial semantic processing. Neuroimage, 53, 638-646

Gusnard, D. A., Akbudak, E., Shulman, G. L., \& Raichle, M. E. (2001). Medial prefrontal cortex and self-referential mental activity: Relation to a default mode of brain function. Proceedings of the National Academy of Sciences, U.S.A., 98, 4259-4264.

Hagoort, P. (2005). On Broca, brain, and binding: A new framework. Trends in Cognitive Sciences, 9, 416-423.

Hagoort, P. (2013). MUC (memory, unification, control) and beyond. Frontiers in Language Sciences, 4, 416.

Hagoort, P., Baggio, G., \& Willems, R. M. (2009). Semantic unification. In M. S. Gazzaniga (Ed.), The cognitive neurosciences IV (pp. 819-836). Cambridge, MA: MIT Press.

Hagoort, P., \& Indefrey, P. (2014). The neurobiology of language beyond single words. Annual Review of Neuroscience, 37, 347-362.

Hamann, S., \& Mao, H. (2002). Positive and negative emotional verbal stimuli elicit activity in the left amygdala. NeuroReport, 13, 15-19.

Herbert, C., Ethofer, T., Anders, S., Junghofer, M., Wildgruber, D., Grodd, W., et al. (2009). Amygdala activation during reading of emotional adjectives-An advantage for pleasant content. Social Cognitive and Affective Neuroscience, 4 , 35-49.

Holt, D. J., Lakshmanan, B., Freudenreich, O., Goff, D. C., Rauch, S. L., \& Kuperberg, G. R. (2011). Dysfunction of a cortical midline network during emotional appraisals in schizophrenia. Schizophrenia Bulletin, 37, 164-176.

Humphries, C., Binder, J. R., Medler, D. A., \& Liebenthal, E. (2007). Time course of semantic processes during sentence comprehension: An fMRI study. Neuroimage, 36, 924-932.

Isenberg, N., Silbersweig, D., Engelien, A., Emmerich, S., Malavade, K., Beattie, B., et al. (1999). Linguistic threat activates the human amygdala. Proceedings of the National Academy of Sciences, 96, 10456-10459.

Jackendoff, R. (2002). Foundations of language: Brain, meaning, grammar, evolution. Oxford, UK: Oxford University Press.

Keuleers, E., Brysbaert, M., \& New, B. (2010). SUBTLEX-NL: A new frequency measure for Dutch words based on film subtitles. Behavior Research Methods, 42, 643-650.

Kim, H., Somerville, L. H., Johnstone, T., Alexander, A., \& Whalen, P. J. (2003). Inverse amygdala and medial prefrontal cortex responses to surprised faces. NeuroReport, 14, 2317-2322.

Kober, H., Barrett, L. F., Joseph, J., Bliss-Moreau, E., Lindquist, K., \& Wager, T. D. (2008). Functional grouping and cortical-subcortical interactions in emotion: A meta-analysis of neuroimaging studies. Neuroimage, 42, 998-1031.

Kuchinke, L., Jacobs, A. M., Grubich, C., Võ, M. L.-H., Conrad, M., \& Herrmann, M. (2005). Incidental effects of emotional valence in single word processing: An fMRI study. Neuroimage, 28, 1022-1032.
Kuperberg, G. R., Lakshmanan, B. M., Caplan, D. N., \& Holcomb, P. J. (2006). Making sense of discourse: An fMRI study of causal inferencing across sentences. Neuroimage, 33, 343-361.

Lamm, C., \& Singer, T. (2010). The role of anterior insular cortex in social emotions. Brain Structure and Function, 214, 579-591.

Lane, R. D., Reiman, E. M., Ahern, G. L., Schwartz, G. E., \& Davidson, R. J. (1997). Neuroanatomical correlates of happiness, sadness, and disgust. The American Journal of Psychiatry, 154, 926-933.

Lang, P. J., Bradley, M. M., \& Cuthbert, B. N. (2008). International affective picture system (IAPS): Affective ratings of pictures and instruction manual. Technical Report A-8. University of Florida, Gainesville.

Luan Phan, K., Wager, T. D., Taylor, S. F., \& Liberzon, I. (2004). Functional neuroimaging studies of human emotions. CNS Spectrums, 9, 258-266.

Maddock, R. J., Garrett, A. S., \& Buonocore, M. H. (2003). Posterior cingulate cortex activation by emotional words: fMRI evidence from a valence decision task. Human Brain Mapping, 18, 30-41.

Menenti, L., Petersson, K. M., Scheeringa, R., \& Hagoort, P. (2009). When elephants fly: Differential sensitivity of right and left inferior frontal gyri to discourse and world knowledge. Journal of Cognitive Neuroscience, 21, 2358-2368.

Moll, J., de Oliveira-Souza, R., Bramati, I. E., \& Grafman, J. (2002). Functional networks in emotional moral and nonmoral social judgments. Neuroimage, 16, 696-703.

Moors, A., De Houwer, J., Hermans, D., Wanmaker, S., van Schie, K., Van Harmelen, A. L., et al. (2013). Norms of valence, arousal, dominance, and age of acquisition for 4,300 Dutch words. Behavior Research Methods, 45, 169-177.

Moseley, R., Carota, F., Hauk, O., Mohr, B., \& Pulvermuller, F. (2012). A role for the motor system in binding abstract emotional meaning. Cerebral Cortex (New York, NY), 22, 1634-1647.

Nakic, M., Smith, B. W., Busis, S., Vythilingam, M., \& Blair, R. J. R. (2006). The impact of affect and frequency on lexical decision: The role of the amygdala and inferior frontal cortex. Neuroimage, 31, 1752-1761.

Nichols, T., Brett, M., Andersson, J., Wager, T., \& Poline, J.-B. (2005). Valid conjunction inference with the minimum statistic. Neuroimage, 25, 653-660.

Novick, J. M., Trueswell, J. C., \& Thompson-Schill, S. L. (2005). Cognitive control and parsing: Reexamining the role of Broca's area in sentence comprehension. Cognitive Affective Behavioural Neuroscicence, 5, 263-281.

Oldfield, R. C. (1971). The assessment and analysis of handedness: The Edinburgh inventory. Neuropsychologia, 9, 97-113.

Poeppel, D., Emmorey, K., Hickok, G., \& Pylkkänen, L. (2012). Towards a new neurobiology of language. Journal of Neuroscience, 32, 14125-14131.

Poline, J. B., Worsley, K. J., Evans, A. C., \& Friston, K. J. (1997). Combining spatial extent and peak intensity to test for activations in functional imaging. Neuroimage, 5, 83-96.

Reiman, E. M., Lane, R. D., Ahern, G. L., Schwartz, G. E., Davidson, R. J., Friston, K. J., et al. (1997). Neuroanatomical correlates of externally and internally generated human emotion. The American Journal of Psychiatry, 154, 918-925.

Rodd, J. M., Davis, M. H., \& Johnsrude, I. S. (2005). The neural mechanisms of speech comprehension: fMRI studies of semantic ambiguity. Cerebral Cortex, 15, 1261-1269.

Rodd, J. M., Johnsrude, I. S., \& Davis, M. H. (2012). Dissociating frontotemporal contributions to semantic ambiguity 
resolution in spoken sentences. Cerebral Cortex, 22, 1761-1773.

Singer, T., Critchley, H. D., \& Preuschoff, K. (2009). A common role of insula in feelings, empathy and uncertainty. Trends in Cognitive Science, 13, 334-340.

Snijders, T. M., Vosse, T., Kempen, G., Van Berkum, J. J. A., Petersson, K. M., \& Hagoort, P. (2009). Retrieval and unification of syntactic structure in sentence comprehension: An fMRI study using word-category ambiguity. Cerebral Cortex, 19, 1493-1503.

Strange, B. A., Henson, R. N. A., Friston, K. J., \& Dolan, R. J. (2000). Brain mechanisms for detecting perceptual, semantic, and emotional deviance. Neuroimage, 12, 425-433.

Tabert, M. H., Borod, J. C., Tang, C. Y., Lange, G., Wei, T. C., Johnson, R., et al. (2001). Differential amygdala activation during emotional decision and recognition memory tasks using unpleasant words: An fMRI study. Neuropsychologia, 39, 556-573.

Taylor, S. F., Welsh, R. C., Wager, T. D., Phan, K. L., Fitzgerald, K. D., \& Gehring, W. J. (2004). A functional neuroimaging study of motivation and executive function. Neuroimage, 21, 1045-1054.

Teasdale, J. D., Howard, R. J., Cox, S. G., Ha, Y., Brammer, M. J., Williams, S. C. R., et al. (1999). Functional MRI study of the cognitive generation of affect. American Journal of Psychiatry, 156, 209-215.
Tzourio-Mazoyer, N., Landeau, B., Papathanassiou, D., Crivello, F., Etard, O., Delcroix, N., et al. (2002). Automated anatomical labeling of activations in SPM using a macroscopic anatomical parcellation of the MNI MRI single-subject brain. Neuroimage, 15, 273-289.

van Ackeren, M. J., Casasanto, D., Bekkering, H., Hagoort, P., \& Rueschemeyer, S. A. (2012). Pragmatics in action: Indirect requests engage theory of mind areas and the cortical motor network. Journal of Cognitive Neuroscience, 24, 2237-2247.

Vytal, K., \& Hamann, S. (2010). Neuroimaging support for discrete neural correlates of basic emotions: A voxel-based meta-analysis. Journal of Cognitive Neuroscience, 22, 2864-2885.

Watson, D. (1988). Intraindividual and interindividual analyses of positive and negative affect: Their relation to health complaints, perceived stress, and daily activities. Journal of Personality and Social Psychology, 54, 1020.

Willems, R. M., Clevis, K., \& Hagoort, P. (2011). Add a picture for suspense: Neural correlates of the interaction between language and visual information in the perception of fear. Social, Cognitive and Affective Neuroscience, 6, 404-416.

Willems, R. M., Ozyurek, A., \& Hagoort, P. (2007). When language meets action: The neural integration of gesture and speech. Cerebral Cortex, 17, 2322-2333. 